\title{
A STUDY ON CODEBOOK DESIGN TECHNIQUES IN SCMA
}

\author{
Madhura $\mathrm{K}^{1}$, Rajeshree Raut ${ }^{2}$ and M. S. S. Rukmini ${ }^{3}$ \\ ${ }^{1,3}$ Dept. of ECE, Vignan's Foundation for Science, Technology, and Research, \\ Guntur Dist., Andhra Pradesh, India \\ ${ }^{2}$ Dept. of ECE, Government College of Engineering, Nagpur Maharashtra, India \\ ${ }^{1}$ madhura.k00@gmail.com, ${ }^{2}$ raut.rajeshree@gmail.com, \\ 3mssrukmini@gmail.com
}

\begin{abstract}
Sparse Code Multiple Access (SCMA) is a NOMA (Non- Orthogonal Multiple Access) technique based on the design of codebook, proposed to manage the varied and demanding performance for massive Machine-Type Communication (m-MTC) in 5G wireless communication systems. In SCMA, to enhance the Bit Error Rate (BER) with other performance parameters, the primary source is the codebook design that is generated based on Mother Constellation. This paper discusses the techniques of designing the codebook and algorithms, using specified Mother Constellation to enhance the bit error rate. It also explains the use of Deep learning for designing a codebook.
\end{abstract}

Keywords - SCMA, Low Density Signature (LDS), NOMA, 5G Communications, Message Passing Algorithm (MPA)

\section{INTRODUCTION}

The requirements of $5 \mathrm{G}$ communications include more substantial connectivity with low latency, including massive data traffic with reduced bit error rate. Orthogonal Frequency Division Multiple Access (OFDMA) is a technique used by $4 \mathrm{G}$ wireless communication which fails to serve these future requirements of $5 \mathrm{G}$ communications, thus leading to the research of various NOMA techniques. As per the comparative study of different NOMA techniques [1] [2], SCMA [3] proves to be the most reliable candidate in NOMA techniques for $5 \mathrm{G}$ communications. SCMA directly maps the incoming bits to complex multi-dimensional codeword selected from predefined codebook sets [4]. This technique is designed from the Low-Density Signature (LDS) [5] based CDMA approach (LDS-CDMA), but has better spectral efficiency and has better shaping gain or coding gain of multidimensional constellation [6]. In this technique, the Quadrature Amplitude Modulation (QAM) mapper and the CDMA spreader are clubbed together, and a set of bits are mapped, directly into a complex codeword, selected from pre-defined codebook set. A specific codebook set has been defined for every layer. These layers are placed non-orthogonally above each other.

At the receiver end the Message Passing Algorithm, MPA detector is used with moderate complexity. The sparseness of code words enables the MPA to alleviate the inter-user interference with reduced complexity. The BER, bit error rate of SCMA is primarily dependent upon the codebooks [8], but designing a codebook is comparatively a more significant problem still under study. The SCMA model with the steps to implement it is shown in Fig.1.

Received: September 21, 2018

Reviewed: December 15, 2018

Accepted: December 21, 2018

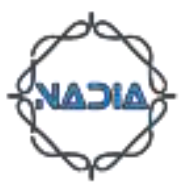


This paper mainly focuses on the second part shown in Fig.1 that is codebook design, and various methods of designing Mother Constellations and codebook to improve the BER and the spectral efficiency.

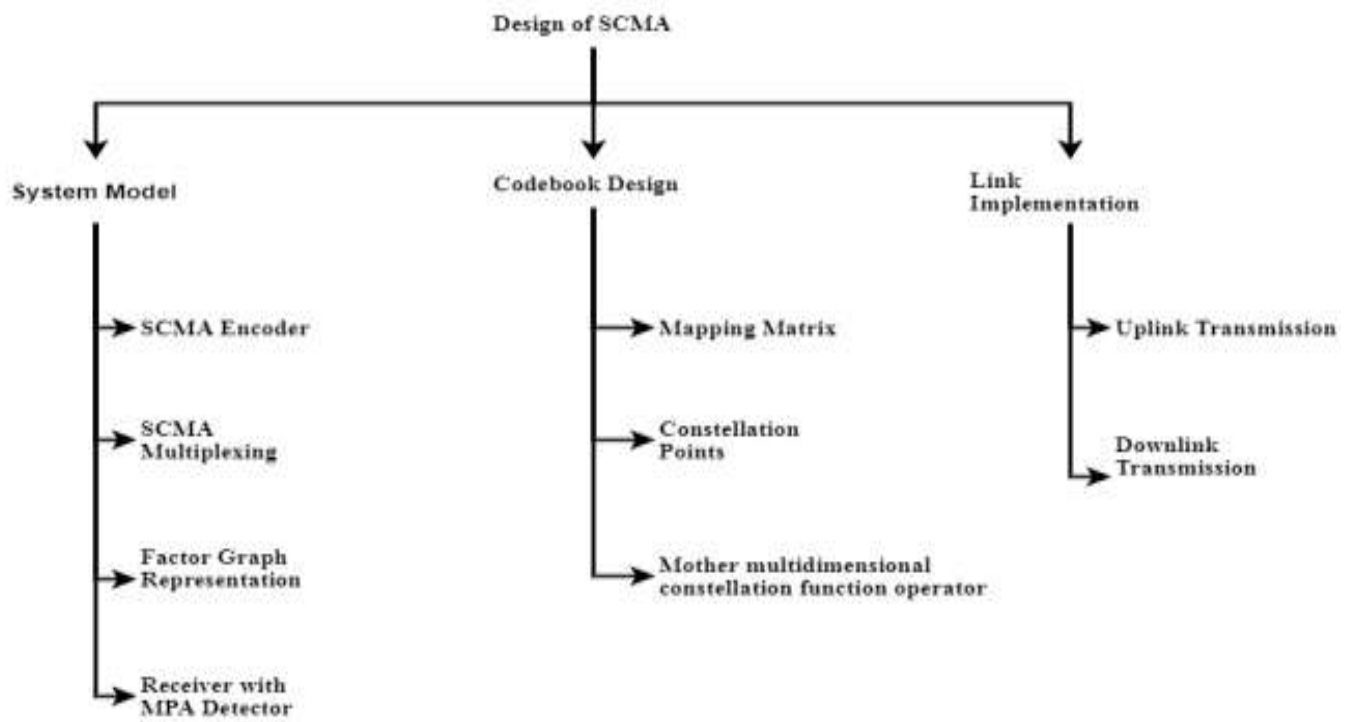

Fig. 1. Designing steps of SCMA $[3,9,10]$

\section{CODEBOOK DESIGN}

The fundamental aim of the codebook design is to maximize the shaping gain by providing good distance properties in the multidimensional constellation. Following steps are involved in designing a codebook.

- To generate multidimensional modulation constellations.

- To transfer the constellation to multiple sparse codebooks.

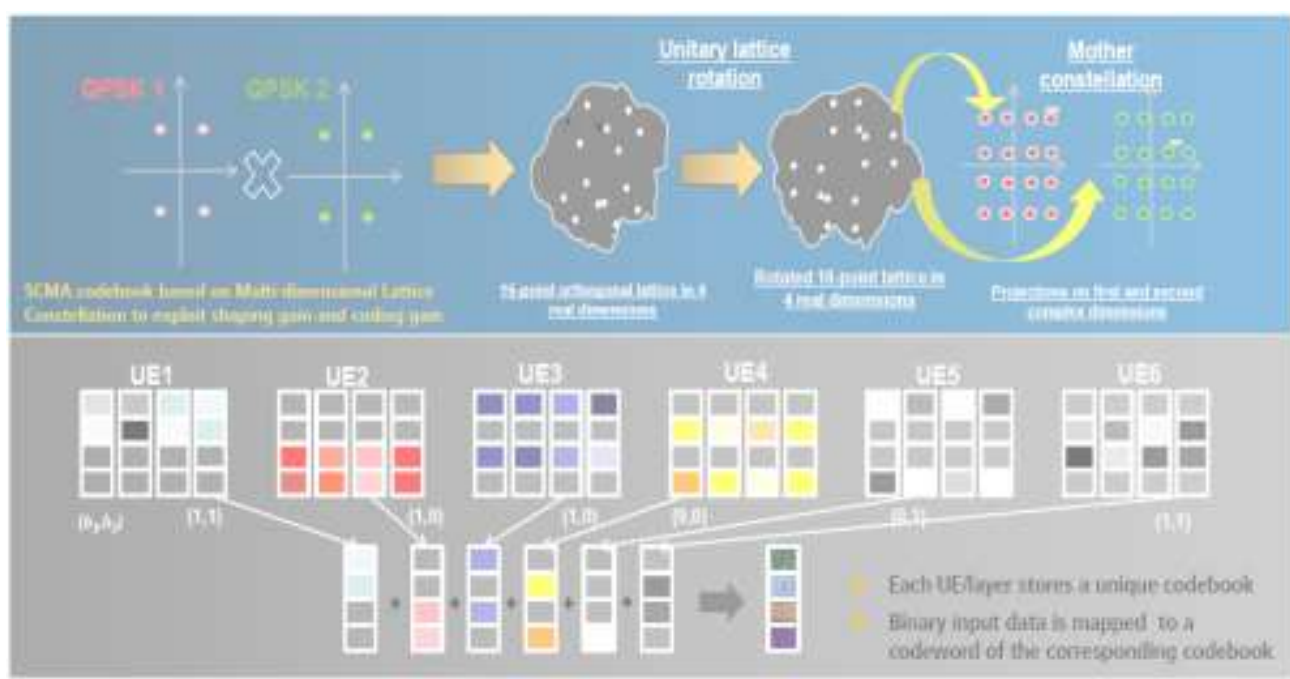

Fig. 2 Codebook Design [11]

We need to define $\mathrm{J}$ different $\mathrm{N}$-dimensional constellation each containing $\mathrm{M}$ points [3]. The complex domain constellation is used as mother constellation after converting it from real domain constellation. Serially concatenated operators applied on mother 
constellation construct the constellation of each layer. Based on the mother constellation and layer-specific operators, the constellation points of layers are modeled. Generally, there are multiple mother constellations, and any of the available mother constellations can build the layer constellation. $g_{j} \equiv\left(\boldsymbol{\Delta}_{j}\right) g, \forall j$ [3], where the constellation operator can be denoted as $\Delta \mathrm{j}$ and $\mathrm{g}$ is denoted as mother constellation. Sub-optimally the mother constellation and the operators need to be determined separately. The conversion from real domain to complex domain does not change the properties of the constellation. As the mother constellation is decided the unitary operations can be performed on the same for the construction of SCMA code. Fig. 2 shows the process of formation of the codebook. It first explains the creation of codebook based on multi-dimensional Lattice constellation. If we have 4 real dimensions, then the lattice constellation will be $2^{4}$, i.e., 16-point orthogonal lattice. The unitary operations (rotations) are performed on the lattice constellation using unitary operators. The real constellations are projected on one another to form a complex constellation. As discussed earlier every layer has its own codebook, the binary input is mapped into codeword from every layer, and they are superimposed upon each other to form a codebook which is transmitted.

\subsection{SCMA CODEBOOK DESIGNING TECHNIQUES}

The design of codebook and mother constellation is responsible for reducing the bit error rate. Following are the methods to improve the same.

2.1.1. DYNAMICALLY CHANGING CODEBOOKS [12]: The codebooks can be shaped with different constellations. According to [13], the dynamically changing codebook reduces bit error at given SNR. As shown in Fig. 3, considering the same parameters from the Tanner graph, we have first layer codebook constellation by changing the angles of the vectors of the first codebook, the other codebooks for the layers can be designed. This fills the complex signal plane and also helps in reducing the strength of the transmitted signal.
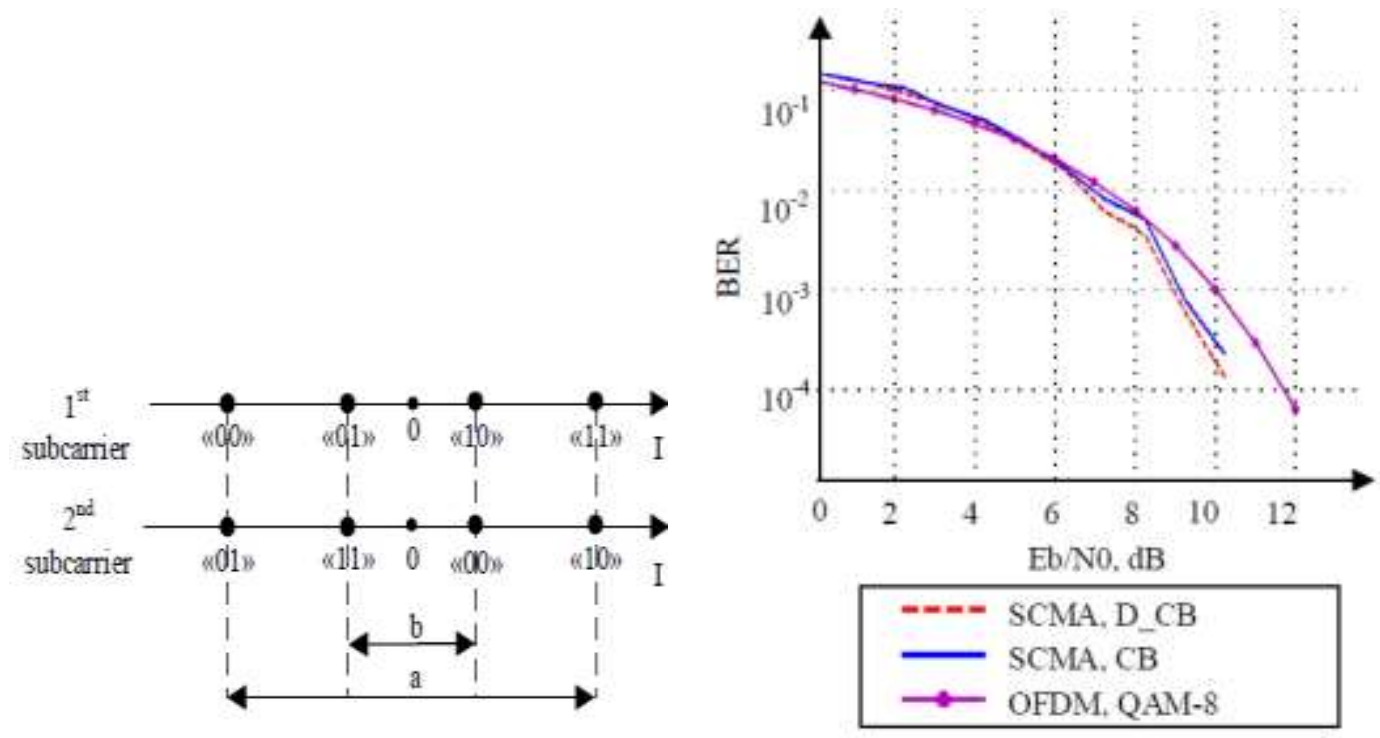

Fig. 3 Single Layer Constellation structure and graph of BER [12]

2.1.2. CODEBOOK DESIGN BASED ON CONSTELLATION ROTATION [14]: Design of Codebook based on constellation rotation has been used for downlink SCMA system. This method explains the concept of main-constellation and sub-constellations. Yongkang Zhou, Qiyue Yu, Weixiao Meng, and Cheng L proposed in [14] to construct 
sub-constellations with proper shape and propose a method to build Latin generator matrices, to build main-constellations with more considerable minimum Euclidean distances along with the introduction of a new model to design codebook. Subconstellation is a 2-D of the main constellation. The main constellation consists of $\mathrm{K}$ complex symbols; the sub-constellation is the $\mathrm{K}^{\text {th }}$ vector of the primary constellation. Figure 4.1 and 4.2 shows different constellations by changing the phase operators using BPSK and QPSK modulation respectively. We have generator matrix G. By introducing appropriate values of non-zero entries in the generator matrix shape of the subconstellation is optimized as shown in Fig. 4.
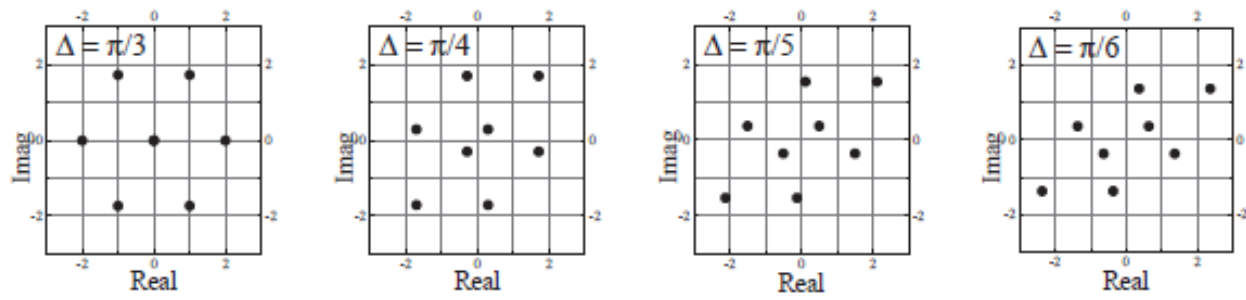

Fig. 4.1 Sub-constellations with different $\Delta$ when using BPSK modulation
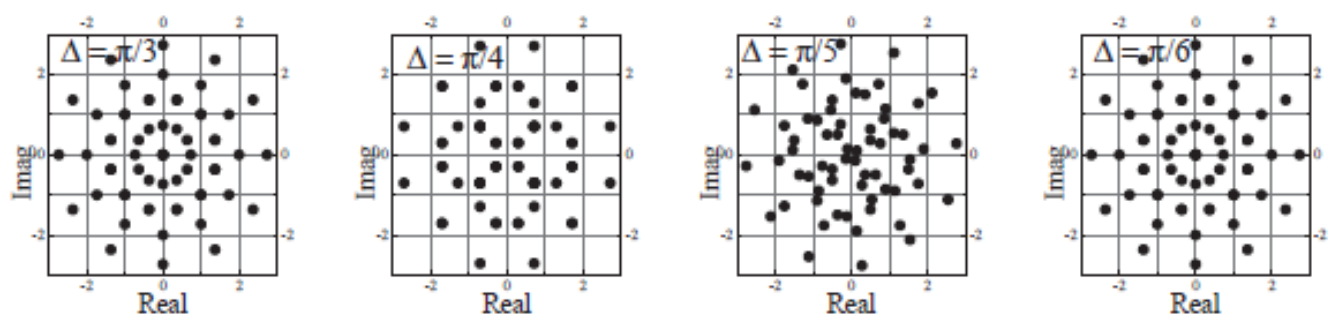

Fig. 4.2 Sub-constellations with different $\Delta$ when using QPSK modulation

It has also proposed, a method to design a latin generator matrix [14] for SCMA codebook. Thus the Fig. 5 shows the graph of BER Vs. Eb/N0 for both BPSK and QPSK obtained by designing the latin generator matrix.

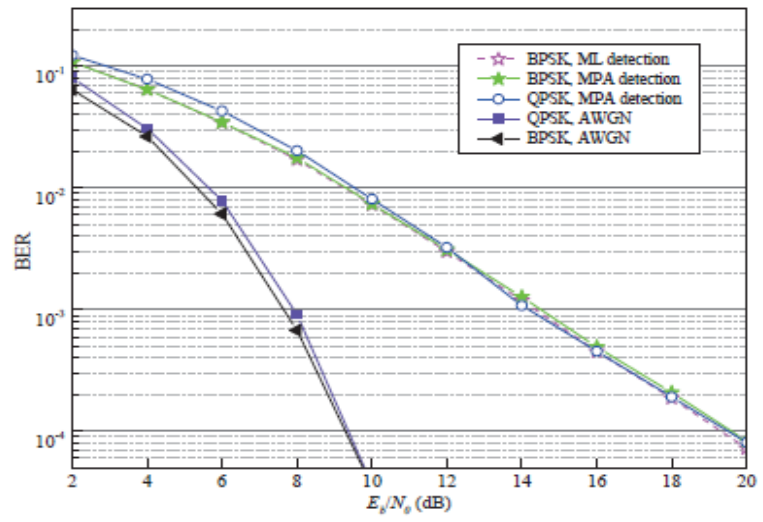

Fig. 5 BER Vs Eb/NO

2.1.3. DESIGN OF MOTHER CONSTELLATION BY MAXIMIZING THE EUCLIDEAN DISTANCE [15]: For improving the minimum Euclidean distance of the Mother Constellation must have the maximum energy or in space. Fig. 6 shows the fourpoint constellation where the points are distributed with maximum possible diversity in both energy and space. For $\mathrm{M}=4 \& \mathrm{~N}=2$, the two vectors have been arranged for having maximum, minimum Euclidean distance given by 


$$
\begin{aligned}
& \mathrm{MC}=\left(\begin{array}{llll}
\mathrm{S}_{1} & \mathrm{~S}_{2} & \mathrm{~S}_{3} & \mathrm{~S}_{4}
\end{array}\right) \\
& =\left(\begin{array}{l}
-3 p_{1}-p_{1}+p_{1}+3 p 1 \\
-p 2-3 p 2+3 p 2+p 2
\end{array}\right)
\end{aligned}
$$

$\alpha$ is responsible for energy diversity and minimum Euclidean distance of Mother Constellation (MC).

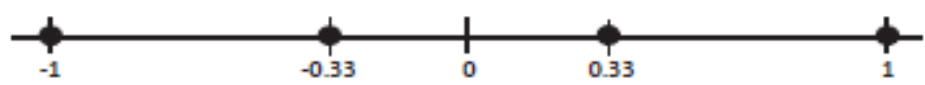

Fig. 6 Constellation point distribution [15]

Now the next step is designing the codebook. The three operations to be performed on the codebook for its generation are:

i) Complex Conjugate

ii) Phase Rotation

iii) Vector Permutation [4]

Here the codebook design for the specific layer can be formulated by the following:

$\mathrm{CB}=\arg \max _{\gamma}(\Delta * \mathrm{~V} * \mathrm{MC})$

In the above formula, $\Delta$ may be designed using either one or the combination of the above operations.

2.1.4. DIMENSION BASED SCMA CODEBOOK DESIGN [16]: This approach uses Turbo TCM Technology to increase the minimum Euclidean distance. Once it is achieved the constellation undergoes phase rotation and coordinates interleaving to increase the number of distinct coordinates between any constellation points and also the coordinate product distance between them. This makes use of pure MPA receiver. Fig. 7 shows the proposed design of the codebook in [16].

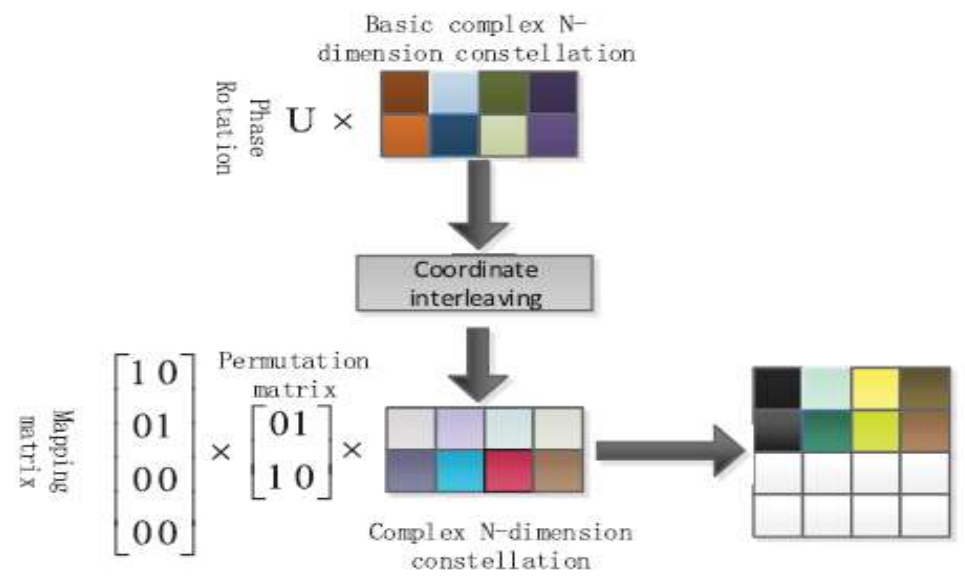

Fig. 7 Proposed SCMA Codebook design [16]

This receiver requires the initial information on each resource node. Hence the permutation sets are first selected that can capture as large as the sum of the distance between dimensions of interfering codewords multiplexed on each resource node. Then to 
design the codebook the constellation and the permutation matrix is combined to increase the distance between dimensions of interfering codewords multiplexed on each resource node. This improves the quality of MPA receiver. The receiver detects the most robust user according to the hierarchy of its strength as shown in Fig. 8.

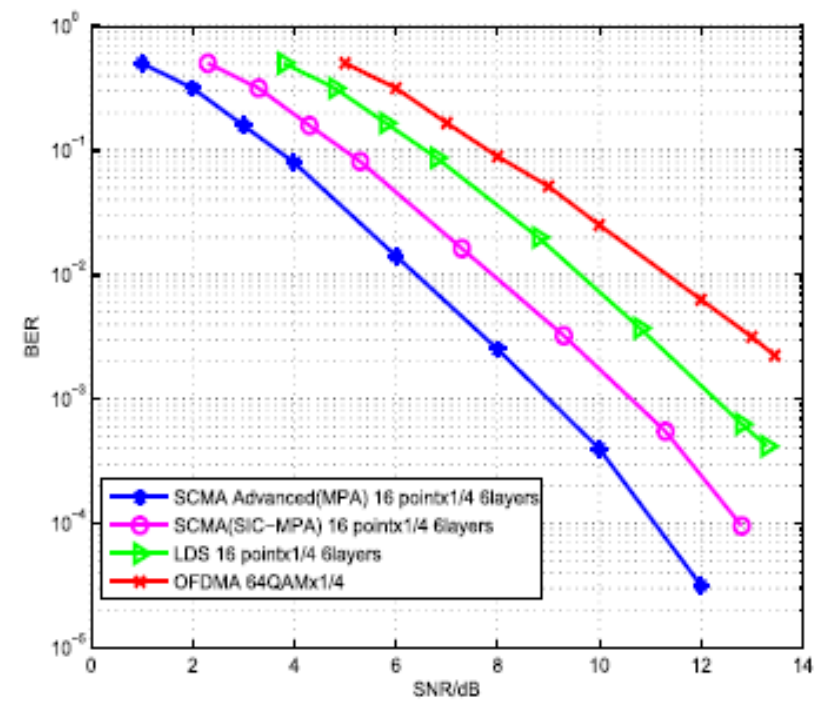

Fig. 8 BER Vs. Eb/N0 of SCMA Advanced with MPA receiver, SCMA with SIC-MPA receiver, LDS and OFDMA system over AWGN channel with the spectral efficiency 1.5 bits/tone. [16]

\subsubsection{SCMA CODEBOOK DESIGN BASED ON CONSTELLATION ROTATION \& INTERLEAVING [17]}

This method of SCMA codebook designing is based on constellation and interleaving. In this, the subset of $2 \mathrm{D}$ is designed for the $1^{\text {st }}$ dimension of $\mathrm{MC}$ while the other dimensions can be obtained by rotating the $1^{\text {st }}$ dimension. Then one to one gray mapping is done to define the codeword, where the elements of even dimensions are interleaved. Constellation operator with specific phase based on Latin structure and factor graph $b$ is designed for the user.

$\mathrm{MC}=(\mathrm{S} 1, \mathrm{~S} 2, \mathrm{~S} 3)$ is designed from the above process where $\mathrm{S}$, is the subset of $16 \mathrm{QAM}$, $\mathrm{S} 2$, and $\mathrm{S} 3$ are obtained by rotating $\mathrm{S} 1$ where $\mathrm{M}=4, \mathrm{~N}=3$, the processes are explained in Fig. 9.

2.1.6. ALGORITHMS FOR DESIGNING CODEBOOK [18]: There are three algorithms described in [18] to enhance the codebook design and improve the BER. The first method is referred to as Fixed User Order (FUO). It starts with the $1^{\text {st }}$ user and maps non-zero elements of its codebook to subcarriers with the best gain, and the procedure is used for the other users as stated in Algorithm 1.

Another algorithm is OA Opportunistic Allocation. In this method, the users are first ordered based on their overall quality of subcarrier gains, and with the help of metric, the user that maximizes it is found, then the codebooks are assigned similar to that in FUO as shown in Algorithm 2.

These two algorithms give the best average BER performance when CSI is not in use, but for an individual user to improve BER, another algorithm is used as stated in the next approach. The third approach studied here is PF, Proportional Fairness which provides a trade-off between performance and fairness. As shown in Algorithm 3. 


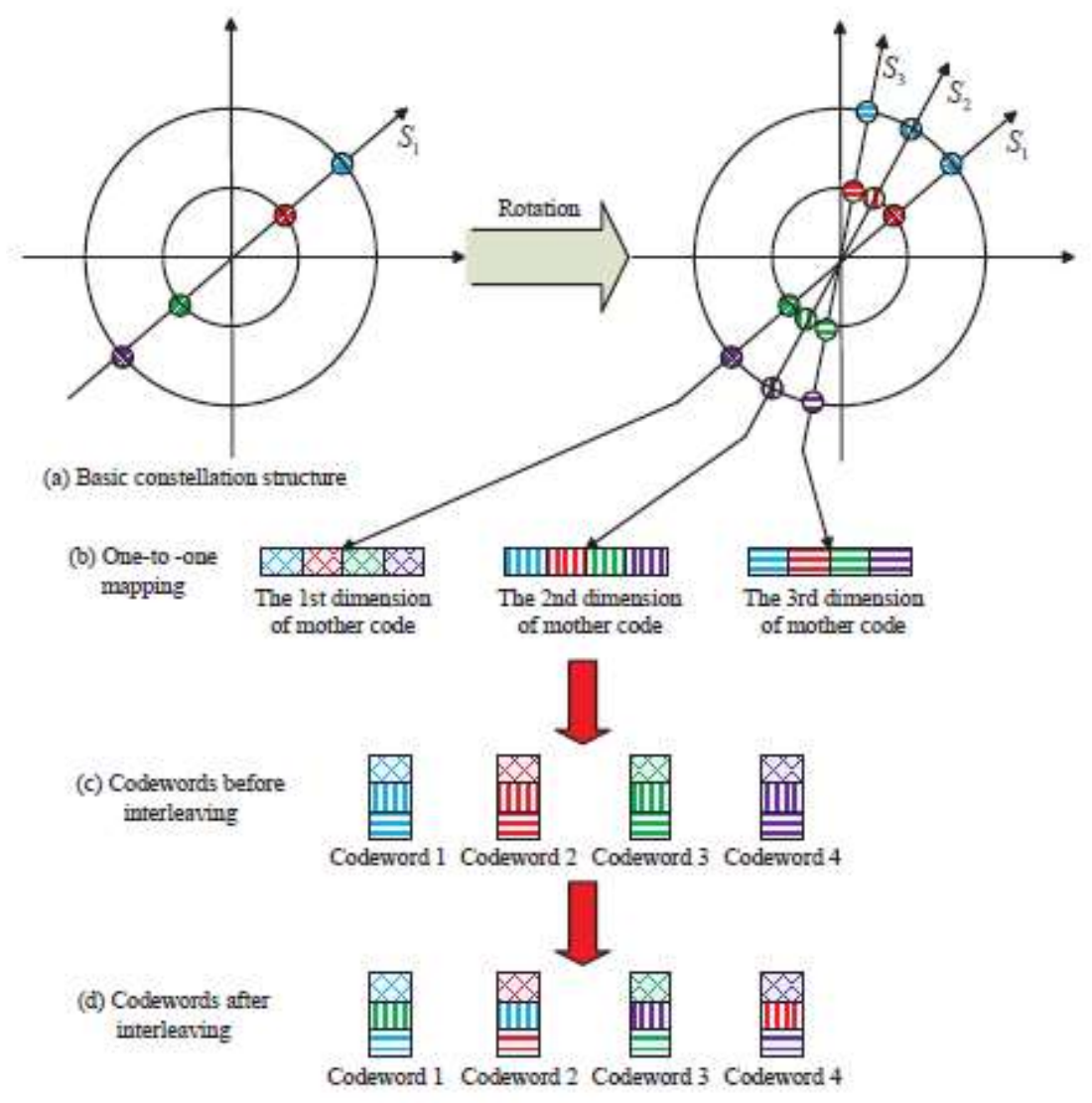

Fig. 9. SCMA Codebook Design [17]

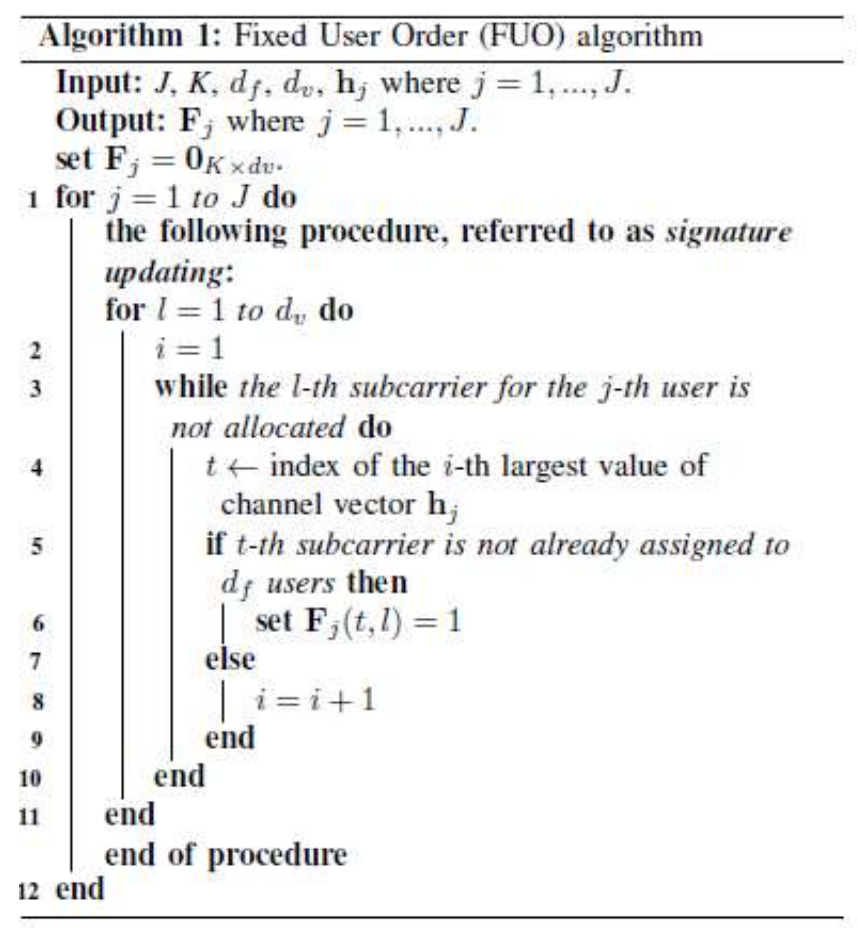

Algorithm 1 [18] 


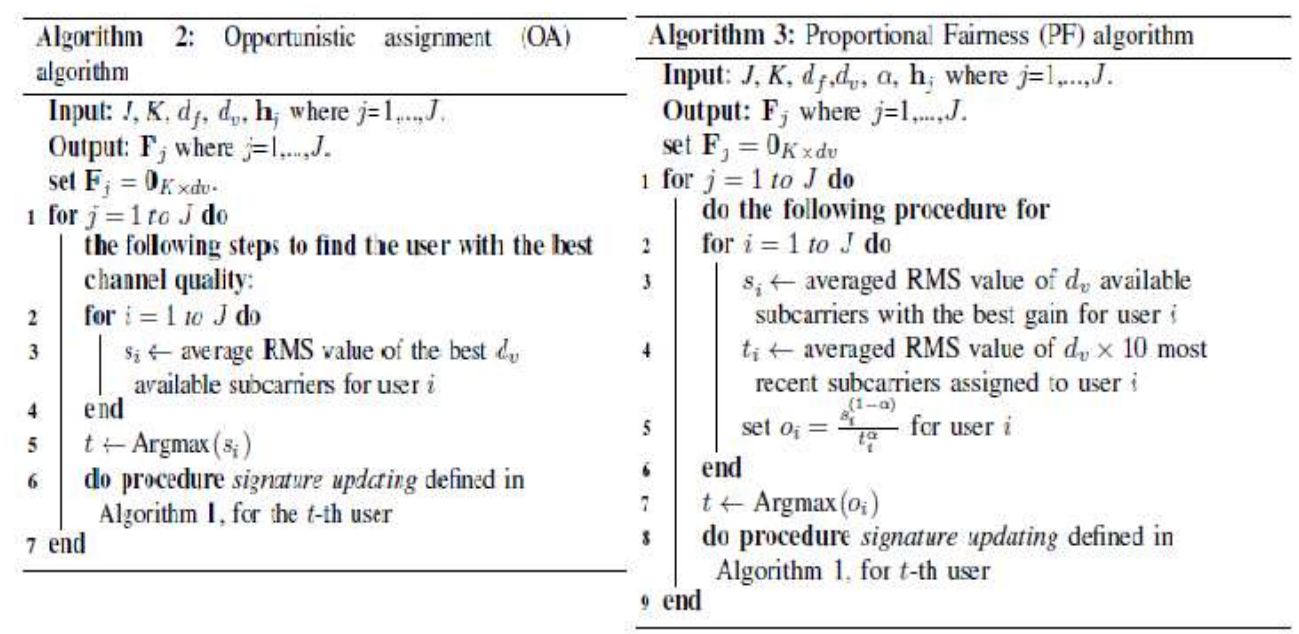

Algorithm 2 and Algorithm 3 [18]

2.1.7. DEEP LEARNING AIDED SCMA [19]: It is also called as D-SCMA. In this method the DNN (Deep Neural Network) helps in finding the optimum method of designing a codebook from input data to the mapping matrix. The M-ary symbol $r_{j}$ is mapped to a complex constellation denoted by $\mathrm{f}_{\mathrm{kj}}(\mathrm{r})$ where a signal source is denoted by $\mathrm{k}$.

Several DNN units are positioned between the streams and resources so as to get the suitable mapping. The units in DNN are set to 6 with 32 hidden nodes. Both, units and nodes together act as codebook generator. The structure of D-SCMA is shown in Fig.11. This outperforms the conventional SCMA in complexity as well as BER [19]. The BER analysis as compared to the convention SCMA is shown in Fig. 10.

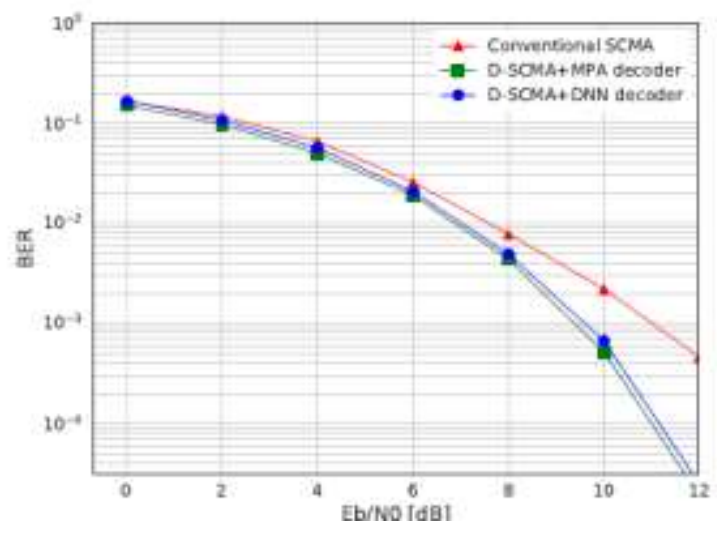

Fig.10 BER analysis of conventional SCMA with DSCMA 


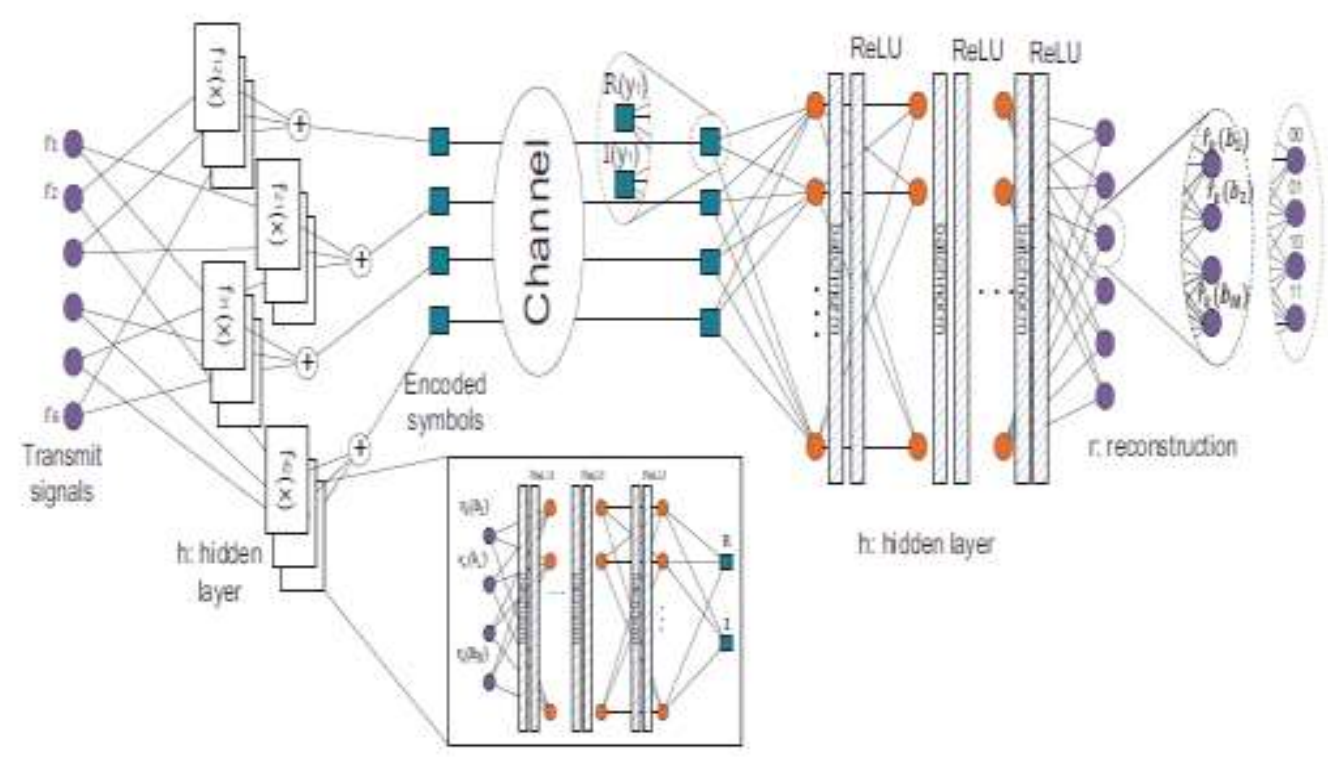

Fig.11 DSCMA Model

\section{LINK IMPLEMENTATION}

By doing minor modifications in the LTE transceiver, the link level simulations for SCMA are implemented. The change made in the transmitter is done by replacing the QAM modulator and the DFT block by SCMA encoder which maps the coded bits into a multidimensional codeword. In the transmitter only by making few modifications in the LTE receiver, the receiver of SCMA is designed by replacing single user channel equalization and QAM de-mapper with SCMA decoder of each layer. The MPA algorithm performs tanner graph constructed by the codebooks. It starts with the initial conditional probability calculation at each function node.

Then it enters MP iterations between the function node and the variable node. For each iteration, both the nodes are updated; this is done independently by each pair. After several sufficient iterations, LLR (Log-Likelihood ratio) for coded bits are calculated based on codeword probability \& output at the variable node and can be given as the input to the turbo decoder.

\section{CONCLUSION}

In this paper, a study of the parameters required to design the SCMA codebook and the up-link and downlink implementation are discussed. The primary motive of any communication model is to enhance the BER performance. To achieve the same, an in depth study of various techniques to design the codebook and the mother constellations required is presented. As seen from all the methods considered in the literature it is concluded that the dynamically changing codebook approach is better one because it helps to improve the BER of the system as shown in Fig. 3. The approach used by the method of Latin generator matrix is also very good at enhancing the BER.. But with the help of deep learning supported by Artificial Intelligence, the communication model will improve several performance parameters jointly. At the end it is concluded that these methods work in high SNR region without sacrificing the system implementation complexity. 


\section{REFERENCES}

[1] M. Kulkarni, R. Raut, "SCMA-An Advancement towards 5G communications", Advanced Science and Technology Letters Vol.147 (SMART DSC-2017), pp.487-498, 2017.

[2] Y. Cai, Z. Qin, F. Cui, G.Y.Li, and J.A.McCann, "Modulation and Multiple Access for 5G Networks", IEEE Communications Surveys \& Tutorials Volume: PP, Issue: 99, v1, [cs.OH], Feb 2017.

[3] H.Nikopour and H. Baligh, "Sparse code multiple access," IEEE 24th PIMRC, 2013, pp. 332-336.

[4] M. Taherzadeh, H. Nikopour, A. Bayesteh and H. Baligh, "SCMA codebook design", IEEE Vehicular Technology Conference (VTC Fall), pp. 1-5, 2014.

[5] R. Hoshyar, F. P. Wathan, and R. Tafazolli, "Novel Low-Density Signature for Synchronous CDMA Systems over AWGN Channel", IEEE Transactions on Signal Processing, 56(4):1616-1626, April 2008.

[6] L.F.Wei G.D.ForneyJr, "Multidimensional constellations. I. Introduction, figures of merit, and generalized cross constellations", IEEE Journal on selected areas in communications in communications, vol. 7, no. 6, pp. I.877-892, 1989.

[7] D. A. Pokamestov. A. Ya. Demidov, Ya. V. Kryukov, E. V. Rogozhnikov, ”Dynamically changing SCMA codebooks", International Siberian Conference on Control and Communications (SIBCON) 978-1-5090-1081-3/17,2017.

[8] C. Bockelmann, N. Pratas, H. Nikopour, K. Au, T. Svensson, C. Stefanovic, P. Popovski, and A. Dekorsy, "Massive machine type communications in $5 \mathrm{~g}$ : physical and mac-layer solutions", IEEE Communications Magazine, 54(9)pp 59-65, September 2016.

[9] M. Jia, L. Wang, Q. Guo, X. Gu, W. Xiang,“ A Low Complexity Detection Algorithm for Fixed UpLink SCMA System in Mission Critical Scenario”, IEEE Internet of Things Journal, Vol X, NO. X, 2017.

[10] Y.Chen, A. Bayesteh, Y. Wu, M.Taherzadeh, D. Chen and J. Ma, S. Han, "SCMA: A Promising Nonorthogonal Multiple Access Technology for 5G Networks",IEEE, 2016.

[11] "The First 5G Innovation Competition", organized by Altera University. [Online].www.innovateasia.com/5g/en/.

[12] D. A. Pokamestov. A. Ya. Demidov, Ya. V. Kryukov, E. V. Rogozhnikov,“'Dynamically changing SCMA codebooks" 2017 International Siberian Conference on Control and Communications (SIBCON) 978-1-5090-1081-3/17, IEEE 2017.

[13] K Au, "Uplink contention based SCMA for 5G radio access", Globecom Workshops Workshops (GC Wkshps), 2014. - IEEE, 2014. - pp. 900- 905, 2014.

[14] Y. Zhou, Q. Yu, W. Meng, and L Cheng, " SCMA Codebook Design Based on Constellation Rotation", IEEE ICC 2017 Wireless Communications Symposium, 2017.

[15] M. Alam, Qi Zhang, "Designing Optimum Mother Constellation and Codebooks for SCMA", IEEE ICC 2017 Wireless Communications Symposium, 2017.

[16] Cheng, G.I. Kang, and N. Zhang, "A Dimension Distance-Based SCMA Codebook Design ", Special Section on Physical and medium access control layer Advances in 5G Wireless Networks, IEEE Access May 2017.

[17] D. Cai, P. Fan, X. Lei, Y. Liu and D.Chen, "Multi-Dimensional SCMA Codebook based on Constellation Rotation and Interleaving", 2016 IEEE 83rd Vehicular Technology Conference (VTC Spring) 2016.

[18] M. Dabiri and H. Saeedi, " Dynamic SCMA Codebook Assignment Methods: A Comparative Study ", Journal of Latex Class Files, Vol. 14, No. 8, Oct 2017.

[19] M.Kim, N Kim W Lee.K. Elissa, D.Cho, “Deep Learning Aided SCMA,” in press submitted to IEEE Communication Letters. 